\title{
Ontological Distinctions between Means-End and Contribution Links in the $i *$ Framework
}

\author{
Renata Guizzardi ${ }^{1}$, Xavier Franch ${ }^{2}$, Giancarlo Guizzardi ${ }^{1}$ and Roel Wieringa ${ }^{3}$ \\ ${ }^{1}$ Ontology \& Conceptual Modeling Research Group, UFES, Brazil \\ \{rguizzardi, gguizzardi\}@inf.ufes.br \\ ${ }^{2}$ Universitat Politècnica de Catalunya (UPC) \\ Barcelona, Spain \\ francheessi.upc.edu \\ ${ }^{3}$ University of Twente \\ Enschede, The Netherlands \\ roelw@cs. utwente.nl
}

\begin{abstract}
The $i^{*}$ framework is a renowned Requirements Engineering approach. This work is part of an ongoing effort to provide ontological interpretations for the $i^{*}$ core concepts. With this, we aim at proposing a more uniform use of the language, in a way that it can be more easily learned by newcomers and more efficiently transferred to industry. Our approach is based on the application of a foundational ontology named UFO, which is used as a semantically coherent reference model to which the language should be isomorphic. In this paper, we focus on the Means-end and the Contribution links. We aim at presenting the community with some possible ontological interpretations of these links, aiming at promoting constructive debate and receiving feedback about the validity of our assumptions.
\end{abstract}

\section{Introduction}

The $i^{*}$ framework [1] is a renowned Requirements Engineering approach that has been alive for almost two decades, continuously attracting new interest both in academia and industry. The community that develops $i *$ is relatively big and these developers, who are geographically dispersed, tend to ascribe different (and sometimes conflicting) meanings to its constructs. The diversity and looseness of the defined variants and extensions could be considered a barrier for one to learn how to use the language as most of the times, it is hard to grasp when a specific construct can and should be used and when it should not (e.g., see empirical study on the is-a construct [2]). It is our belief that this hampers the efficient communication of knowledge among experts of the community [3], the learning curve of newcomers, and the adoption of the framework by practitioners.

In the past few years, the community has become aware of this problem and several attempts have been made for facilitating the access and uniform use of the $i^{*}$ language. Works on metamodeling have tried to make it clear the meaning ascribed to the distinct constructs [4][5][6]. Although we recognize there are significant outcomes

Guizzardi, R. [et al.]. Ontological distinctions between means-end and contribution links in the $\mathrm{i}^{\star}$ framework. A: International Conference on Conceptual Modeling. "Conceptual Modeling: 32th International Conference, ER 2013, Hong-Kong, China, November 11-13, 2013: proceedings". Hong Kong: Springer, 2013, p. 463-470.

The final authenticated version is available online at https://doi.org/10.1007/978-3-642-41924-9_39 
of these works (e.g. pointing out the applied concepts in particular variations; showing the author's view on how concepts relate), these attempts did not quite succeed in providing interoperability, simply because metamodels are powerful structures to define a language's syntax while being very limited in terms of clarifying its semantics. Cares [7] has proposed an interoperability method that considers a supermetamodel [8], which facilitates the translation from an $i^{*}$ variant to another, and an XML-based mark-up language, named iStarML [9], which triggers existing tools to interoperate as much as their underlying metamodel allows. This approach has advanced the state of the art, by providing a standard interoperability format that facilitates model translation, but we are afraid that iStarML only makes syntactic checks, leaving the semantic interoperability issues still untouched.

Our approach goes beyond the work of Cares, aiming at defining a common ontology for the core concepts of the language. In this paper, our focus is to propose distinctions between the Means-end and the Contribution links, often present in in different languages with closely related but ambiguous meanings. It is our goal to present the community with some possible ontological interpretations of these distinctions, aiming at promoting constructive debate and receiving feedback about the validity of our assumptions.

Ontologies have been used to establish a common understanding regarding a domain of interest, functioning as an interchange language for communication between applications and organizations. Guizzardi [10] proposes a method to evaluate and (re)engineer modelling languages. Such method has been applied in the context of the $i^{*}$ framework [10][12]. It consists on using an ontology as a reference model and trying to make the metamodel of the language isomorphic to this ontology. In this way, the language is said to represent well the domain described by the ontology.

In this work, we apply the UFO ontology [10,11] as the reference model. Our choice for UFO was motivated by our in depth knowledge of its foundations, but also by the substantial record of its successful use to analyze, redesign and integrate languages and reference models in a large number of domains. These analysis initiatives have targeted significant approaches in the literature such as BPMN, ARIS, REA, ITIL, RM-ODP, AORML, UML, Archimate, among others. In particular, in a number of publications, we have used UFO to make explicit the ontological semantics underlying $i^{*}$ and TROPOS $[11,12]$. Finally, in the overall research project of analyzing $i^{*}$, it is fundamental be able to count on a foundational ontology that elaborates on ontological distinctions among object types and that embeds a fuller ontological theory of relations. This requirement makes UFO a more suitable choice for this purpose than alternatives such as GFO and DOLCE.

The remainder of this paper is organized as follows: Section 2 explains the concepts of the UFO foundational ontology which form the basis for the new ontological definitions described in this paper; Section 3 proposes the formal ontological definitions of the Means-end and Contribution links; at last, section 4 discusses whether and if so, what we have contributed to the goal of making $i^{*}$ more usable and useful. 


\section{The Supporting UFO Concepts}

Before analyzing the $i^{*}$ Means-end (ME) and Contribution links, it is important to provide an ontological view of the language intentional elements. We do that by analyzing the language elements in terms of the ontological distinctions put forth by the foundational ontology UFO [11][12].

In UFO, a stakeholder is represented by the Agent concept, defined as a concrete Endurant (i.e. an entity that endures in time while maintaining its identity) which can bear certain Intentional states. These intentional states include Beliefs, Desires and Intentions. Intentions are mental states of Agents which refer to (are about) certain Situations in reality. Situations are snapshots of reality which can be understood as a whole and which can be actual or counterfactual. If a Situation is actual in a certain Time Interval, we say that the situation obtains-in that time interval. Situations are composed of Endurants. In particular, a situation can be composed of sub-situation. If a Situation obtains-in a certain Time Interval then all its constituents exist in that Time Interval.

Intentions have propositional-contents that can be true according to the way the world happens to be (i.e., which Situations actually obtain-in the world in a certain Time Interval). For instance, suppose that I believe that London is the capital of England. This Belief is correct if its propositional-content is true which, in turn, is the case iff there is a city called London and a country called England and the former bears a legal relation of being the capital of to the latter. The propositional-content (i.e., proposition) of an Intention is termed a Goal.

We here take propositions to be abstract ontological entities that bear a relation of satisfiability to situations in the world. Thus, when writing satisfies $(s, p)$ we mean that $p$ is true iff the Situation $s$ obtains-in reality. We also define the predicate implies $\left(p, p^{\prime}\right)$ holding between propositions meaning: (1) implies $\left(p, p^{\prime}\right) \leftrightarrow(\forall s$ Situation $(s) \wedge \operatorname{satisfies}(s, p) \rightarrow$ satisfies $\left.\left(s, p^{\prime}\right)\right)$. The set of situations which satisfy a Goal $\mathrm{G}$ is termed G's satisfiability set.

In contrast to Endurants, Events are perduring entities, i.e., entities that occur in time, accumulating their temporal parts. Events are triggered-by certain Situations in reality (termed their pre-situations) and they change the world by producing a different post-situation. Actions are Events deliberately performed by Agents in order to fulfil their Intentions. So, we state that if an Intention $i$ of Agent $X$ is the reason for the performance of Action $a$ (by $X$ ) then $X$ believes that $a$ has as post-situation $S$ which satisfies the propositional-content of $i$. In case $X^{\prime}$ s belief is correct then we say that Action $a$ achieves Goal $G$, i.e.: (2) achieves $(a, G) \leftrightarrow$ satisfies(post-situation $(a), G)$. In other words, an Action achieves a Goal if the Action brings about a Situation in the world which satisfies that Goal.

We here interpret $i^{*}$ goals, tasks and agents as their counterparts in UFO (with Action as task). As such, we interpret the so-called goal and-decomposition and goal or-decomposition in $i^{*}$ as follows: (3) a goal $\mathrm{G}$ is and-decomposed in goals $\mathrm{G}_{1} \ldots \mathrm{G}_{n}$ iff $\mathrm{G}$ is satisfied by exactly those situations which satisfy $\mathrm{G}_{1} \ldots \mathrm{G}_{n}$ conjunctively, i.e., the situations which satisfied all $\mathrm{G}_{1} \ldots \mathrm{G}_{n}$; (4) a goal $\mathrm{G}$ is or-decomposed in goals 
$\mathrm{G}_{1} \ldots \mathrm{G}_{n}$ iff $\mathrm{G}$ is satisfied by exactly the situations which satisfy the goals $\mathrm{G}_{1} \ldots \mathrm{G}_{n}$ disjunctively, i.e., by any situation which satisfy each of the goals $\mathrm{G}_{1} \ldots \mathrm{G}_{n}$. In the formulae above, we assume that goals $\mathrm{G}_{1} \ldots \mathrm{G}_{n}$ are distinct and all of these goals are informative (i.e., they are possibly achieved).

\section{Means-End Links vs. Contribution Links}

This section is dedicated to provide ontological interpretations for Means-end links and Contribution links in $i^{*}$, especially by comparing and contrasting each other. To distinguish the four Contribution values, we chose to use the GRL syntax of Make and Break (also present in the $i^{*}$ wiki), because these names express more clearly the semantic distinctions among the values, when compared to the traditional $i^{*}$ values of ++ and --. The analysis of the partial contribution links (Help and Hurt) remains as future work. Figure 1 presents two cases which will assist us in the definitions of the links, presented in the following two subsections.

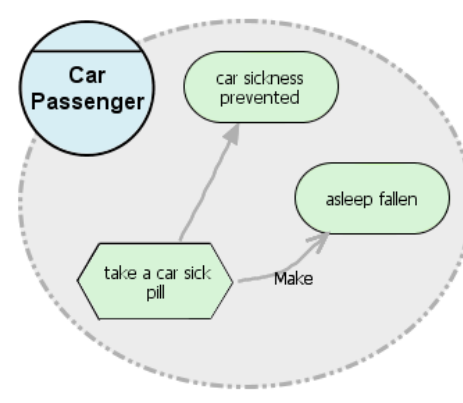

(A)

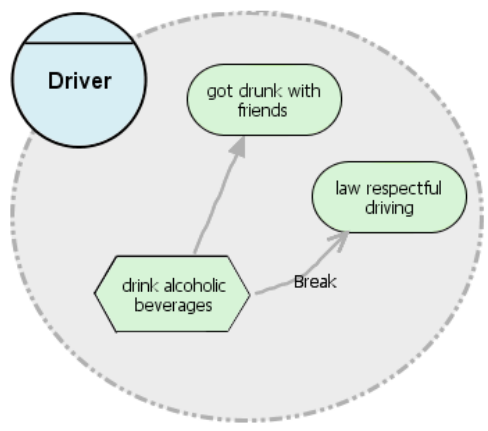

(B)

Fig.1. Examples in $i^{*}$ used to differentiate the Means-end and Contribution links

\subsection{Means-end link vs. Make Contribution link}

Figure 1A) shows an example, in which a Car Passenger ${ }^{1}$ agent executes the take a car sick pill task in order to prevent himself from being sick during the journey he is making (Means-end link to car sickness prevented goal). As a side effect, the Car Passenger also goes to sleep (Make Contribution link to asleep fallen goal).

Both goals depicted in the model are equally accomplished: following the proposal in [12], we here assume that the Means-end link leads to full accomplishment and the Contribution link value is Make, which also indicates the goal is completely fulfilled. So then, what is the distinction among these two links? We here claim that the difference is given by the Intention behind the execution of the task.

\footnotetext{
${ }^{1}$ From now on, we use a different font for the names of the instances of the $i^{*}$ actors and intentional concepts, such as goals, tasks, and resources.
} 
As shown in Section 2, as result of the mapping from $i *$ tasks into UFO actions, every task is associated with a causing intention whose propositional content is a goal. In other words, we execute a particular task in order to accomplish a specific goal. In $i^{*}$, the association between the task and the goal in this case is made by a Means-end link (e.g. take a car sick pill task as means to car sickness prevented goal). On the other hand, this same task can also generate some other goals to be accomplished, without however, being intended by the choice of this particular task. In this case, a Make Contribution link is established (e.g. take a car sick pill task as means to asleep fallen goal). From here on, we focus on a few logical statements that help defining these notions. Firstly, we define the notion of deliberately achieving a goal as follows: (5) deliberately-achieves $(a, G) \leftrightarrow \operatorname{achieves}(a, G) \wedge(\exists i$ intention $(i) \wedge$ isreason-for $(i, a) \wedge$ implies(propositional-content $(i), G)$ ).

In other words, an action $a$ deliberately achieves a goal $G$ iff this action achieves $G$ (i.e., causes the world to be in a state which makes $G$ true) but also this action must be motivated by an intention (intention $i$ is the reason for action $a$ ) whose propositional content implies $G$ (in the sense defined in Section 2). By using this notion, we clarify the distinction between Means-end links (ME) and Make Contribution links: (6) $\operatorname{action}(a) \wedge \operatorname{goal}(G) \wedge M E(a, G) \rightarrow$ deliberately-achieves $(a, G)$; (7) action $(a) \wedge$ $\operatorname{goal}(G) \wedge \operatorname{MakeCont}(a, G) \rightarrow \operatorname{achieves}(a, G) \wedge \neg$ deliberatively-achieves $(a, G)$.

Now, it is important to analyze what are the changes in these relations for the case of having softgoals as ends. To differentiate between a goal and a softgoal, UFO considers the relation of satisfaction as a ternary relation that can hold between an Agent, a Goal and a goal satisfiability set. An instance of this relation is derived from the consideration (Belief) of an agent that a particular set of situations satisfies the goal at hand. In fact, it is exactly this concept which seems to capture the aforementioned notion of softgoals and its difference w.r.t. hardgoals: a goal G is said to be a softgoal iff it is possible that two rational agents $\mathrm{X}$ and $\mathrm{Y}$ differ in their beliefs to which situations satisfy that goal. In other words, we say that a softgoal is satisfied according to the Belief of a particular Agent, while hardgoals are either satisfied or not, with no need to consider the agent or her belief [12].

Following the analysis of the given example, what if instead of a hardgoal, Figure 1A) presented a softgoal, e.g. a feel well softgoal instead of the car sickness prevented hardgoal. Then the analysis would be in place. Firstly, for the case of softgoals, the predicate satisfies must be agent-indexed, i.e., it must be defined as a ternary predicate satisfies $(a, G, X)$. We can then redefine the predicates achieves and deliberatively-achieves for the case of a softgoal. Both redefined predicates now also take into account a third argument, namely, an agent X: (8) achieves $(a, G, X) \leftrightarrow$ satisfies(post-situation $(a), G, X) ;(9)$ deliberatively-achieves $(a, G, X) \leftrightarrow$ achieves $(a$, $G, X) \wedge(\exists i \operatorname{intention}(i) \wedge i$ s-reason-for $(i, a) \wedge \operatorname{implies}(\operatorname{prop}-\operatorname{cont}(i), G))$. The relation of Means-End links is then redefined accordingly: (10) action $(a) \wedge \operatorname{softgoal}(G) \wedge$ $\operatorname{agent}(X) \wedge \operatorname{ME}(a, G, X) \rightarrow$ deliberatively-achieves $(a, G, X)$. Finally, supposing the softgoal was the contribution target (e.g. a got asleep fast softgoal instead of the asleep fallen hardgoal), a similar definition can now be provided for the case of Make Contributions: (11) action $(a) \wedge \operatorname{softgoal}(G) \wedge \operatorname{agent}(X) \wedge \operatorname{MakeCont}(a, G, X) \rightarrow$ achieves $(a, G, X) \wedge \neg$ deliberatively-achieves $(a, G, X)$ 
In other words, when we deal with softgoals as ends, both in the case of Means-end and Contribution, the only existing distinction regards the fact that satisfiability sets for goals are indexed to particular agents.

Table 1 summarizes the difference between ME and Make Contribution links.

Table 1. Guidelines: Means-end link vs. Make Contribution link

- $\quad$ Action $a$---means-end- $\rightarrow$ hardgoal $\mathrm{G}$ for an actor A iff

1. By choosing to perform $a$, it was A's intention to achieve goal $\mathrm{G}$,

2. Performing $a$ causes situation $\mathrm{S}$ and

3. Situation $\mathrm{S}$ satisfies $\mathrm{G}$,

- $\quad$ For softgoal G, replace 2 and 3 by

2. Performing $a$ causes situation $\mathrm{S}$ according to $\mathrm{A}$ and

3. Situation $\mathrm{S}$ satisfies $\mathrm{G}$ according to $\mathrm{A}$

- $\quad$ Action $a$--- make contribution $\rightarrow$ hardgoal $\mathrm{G}$ for an actor A iff

1. By choosing to perform $a$, it was NOT A's intention to achieve goal G,

2. Performing $a$ causes situation $\mathrm{S}$ and

3. Situation $\mathrm{S}$ satisfies $\mathrm{G}$,

- $\quad$ For softgoal G, replace 2 and 3 by

2. Performing $a$ causes situation $\mathrm{S}$ according to $\mathrm{A}$ and

3. Situation $\mathrm{S}$ satisfies $\mathrm{G}$ according to $\mathrm{A}$

\subsection{Break Contribution}

The example of Figure 1B) presents the following situation: a Driver agent drinks a high dosage of alcohol (drink alcoholic beverages task) in order to got drunk with friends. However, this contributes negatively (break-valued contribution link) to his other goal to law respectful driving.

In UFO, a situation triggers an action if the obtaining of that situation enables the action to occur. In other words, the Situation is the pre-situation of that Action. We say that a situation $\mathrm{S}$ disables an action $a$ if that situation conflicts with the situation S' which triggers $a$ (the pre-situation of $a$ ). Having these definitions at hand, we are able to define break contribution. Now, we have also the notion of conflicting situations which can be characterized as follows: (12) $\operatorname{conflicts}\left(S, S^{\prime}\right) \rightarrow(\forall t$ time-interval $(t) \rightarrow \neg\left(\right.$ obtains-in $\left.(S, t) \wedge \operatorname{obtains-in}\left(S^{\prime}, t\right)\right)$. In other words, two situations conflict if they cannot obtain in the same time. We say that a situation $\mathrm{S}$ disables an action $a$ if that situation conflicts with the situation $S^{\prime}$ which triggers $a$ (the presituation of $a)$ : (13) disable $(S, a) \leftrightarrow\left(\forall S^{\prime} \operatorname{triggers}\left(S^{\prime}, a\right) \rightarrow \operatorname{conflicts}\left(S, S^{\prime}\right)\right)$. Finally, we can define the break contribution (BreakCont) relation between an action and a goal as follows: (14) action $(a) \wedge \operatorname{goal}(G) \wedge \operatorname{BreakCont}(a, G) \rightarrow \exists S$ Situation $(S) \wedge$ $(\operatorname{post-\operatorname {situation}}(a)=S) \wedge\left(\forall a^{\prime} \operatorname{Action}\left(a^{\prime}\right) \wedge \operatorname{achieves}\left(a^{\prime}, G\right) \rightarrow \operatorname{disables}\left(S, a^{\prime}\right)\right)$.

In other words, we state that an action $a$ breaks a goal $\mathrm{G}$ iff that action brings the world to a state such that while that state persists, no action which can bring about $\mathrm{G}$ can possibly be performed. In reality, if a situation like the one depicted in Figure 3 is 
modeled in $i^{*}$, then the action represented by the drink alcoholic beverages task disables all actions which would lead to the law respectful driving, i.e., while the situation created by that action persists (the person at hand being drunk), one cannot perform any action of driving which satisfies the goal of driving legally.

Again, we remind the reader that if the end of the contribution were a softgoal, the only distinction regarding the BreakCont definition would be adding the agent in the loop, as follows: (15) action $(a) \wedge \operatorname{goal}(G) \wedge$ agent $(X) \wedge \operatorname{BreakCont}(a, G, X) \rightarrow \exists S$ Situation $(S) \wedge($ post-situation $(a)=S) \wedge\left(\forall a^{\prime} \operatorname{Action}\left(a^{\prime}\right) \wedge \operatorname{achieves}\left(a^{\prime}, G, X\right) \rightarrow\right.$ $\left.\operatorname{disables}\left(S, a^{\prime}\right)\right)$.

Table 2 presents some guidelines for the use of the Break Contribution link.

Table 2. Guidelines for the use of the $i *$ break contribution link

- Action $a$--- break contribution $\rightarrow$ hardgoal $\mathrm{G}$ for an actor A iff

1. Performing $a$ causes situation $\mathrm{S}$ and

2. S disables any action a' that satisfies $\mathrm{G}$.

- $\quad$ For softgoal G, replace 2 by

2. S disables any action a' that satisfies $\mathrm{G}$ according to $\mathrm{A}$.

\section{Conclusions}

In this paper, we report the latest results of a long term research effort in creating a common ontology for the $i^{*}$ core elements. With this work, we hope to contribute to the ongoing effort of the $i^{*}$ community to clarify the specific uses for each of the framework's constructs, as well as to promote interoperability among the distinct $i^{*}$ variants. In [10] we investigated the semantics of the $i^{*}$ intentional elements (e.g. hardgoal, softgoal, resource, etc.) while in [12] we focused in understanding the difference between the Means-end link and the decomposition relations. In this particular paper, we presented ontological distinctions between the Means-end and Contribution links, having actions as means and goals and softgoals as ends. For that, we analyzed these links in light of the UFO foundational ontology. For each analysis made, we provided illustrative examples and logical statements to formalize our findings. Relevant results of this initiative are both an understanding of the involved constructs and some knowledge that $i^{*}$ modelers can use as rationale when constructing their diagrams. However, it is clear that a proposal of this nature cannot be limited to the initiative of particular researchers. A fundamental part of our agenda is to promote the discussion of the ontology foundation of $i^{*}$ at the community level.

From a scientific perspective, the next step of this work is to switch the means and the ends of the investigated links, considering other types of intentional elements as means and ends. In fact, in the logical statement of this paper, we type the means and the ends (e.g. action(A), goal(G), etc.) as an attempt to make these formulas as flexible as possible, having this next step in mind. And in addition to completing the analysis started here, investigating the remaining $i^{*}$ links is also part of our research agenda. 
Acknowledgements. This work has been partially supported by the Spanish project TIN2010-19130-C02-00. We are also grateful to the support provided by FAPES (PRONEX \#52272362/2011) and CNPq Productivity Grant \#311578/2011-0). Finally, we thank Lidia López for her help in designing the figures presented in this paper.

\section{References}

1. Yu, E.: Modeling Strategic Relationships for Process Reengineering. PhD thesis, University of Toronto, Canada (1995).

2. López, L., Franch, X., Marco, J.: Specialization in $i^{*}$ Strategic Rationale Diagrams. ER 2012.

3. López, L., Franch, X., Marco, J.: Making Explicit some Implicit $i^{*}$ Language Decisions. ER 2011.

4. Amyot, D., Horkoff, J., Gross, S. and Mussbacher, G.: A Lightweight GRL Profile for $i *$ Modeling. ER Workshops, LNCS 5833, 254-256 (2009)

5. Susi, A., Perinni, A., Mylopoulos, J. and Giorgini, P.: The Tropos Metamodel and its Use, Informatica, 29, 401-408 (2007)

6. Lucena, M., Santos, E., Silva, C., Alencar, F., Silva, M.J. and Castro, J.: Towards a Unified Metamodel for i*. IEEE RCIS 2008, 237-246 (2008)

7. Cares, C.: From the i* Diversity to a Common Interoperability Framework. PhD Thesis, Polytechnic University of Barcelona, Spain (2012)

8. Wachsmuth G.: Metamodel Adaptation and Model Co-adaptation. LNCS, vol. 4609, pp. 600--624 (2007)

9. Cares C., Franch X., Perini A. and Susi A.: Towards $i *$ Interoperability using iStarML. Computer Standards and Interfaces, 33, 69--79 (2010)

10. Guizzardi, G.: Ontological Foundations for Structural Conceptual Models. Phd Thesis, University of Twente, The Netherlands (2005)

11. Guizzardi, R., Guizzardi, G.: Ontology-based Transformation Framework from Tropos to AORML. In: Yu, E., Giorgini, P., Maiden, N., Mylopoulos, J. (Eds.). Social Modeling for Requirements Engineering, pp. 547-570. MIT Press, Cambridge, MA (2011).

12. Guizzardi, R., Franch, X., Guizzardi, G. Applying a Foundational Ontology to Analyze Means-End Links in the $i *$ Framework. In: 6th IEEE International Conference on Research Challenges in Information Science, pp. 333-343. IEEE Press (2012).

13. Cares, C., Franch, X.: A Metamodelling Approach for $i^{*}$ Model Translations. CAiSE 2011. 